Euskal ikerketen aldizkaria | Revue d'études basques |

Revista de estudios vascos | Basque studies review

2| 1997

Numéro II

\title{
L'orient comme virtualite dans le carnet de 1835 d'Antoine d'Abbadie
}

\section{Aurélie Arcocha-Scarcia}

\section{OpenEdition}

\section{Journals}

Édition électronique

URL : http://journals.openedition.org/lapurdum/1788

DOI : 10.4000/lapurdum. 1788

ISSN : 1965-0655

\section{Éditeur}

IKER

Édition imprimée

Date de publication : 1 octobre 1997

Pagination : 83-91

ISBN : 2-84127-142-0

ISSN : $1273-3830$

Référence électronique

Aurélie Arcocha-Scarcia, «L'orient comme virtualite dans le carnet de 1835 d'Antoine d'Abbadie », Lapurdum [En ligne], 2 | 1997, mis en ligne le 01 septembre 2010, consulté le 30 janvier 2020. URL : http://journals.openedition.org/lapurdum/1788; DOI : 10.4000/lapurdum.1788 


\title{
L'ORIENT COMME VIRTUALITE DANS LE CARNET DE 1835 D'A. D'ABBADIE
}

\begin{abstract}
“Un homme fait le projet de dessiner le Monde. Les annees passent : il peuple une surface dinages de provinces, de rovaumes, de montagnes, de golfes, de navires. d'illes. de poissons. de maisons, d'instruments. d'astres. de chevaux, de gens. Peu avant sa mort. il s apercoit que ce patient lalnrinthe de formes n'est rien d'autre que son portrait.
\end{abstract}

(Borges)

A. Abbadie, en cette année 1835, ne tient pas seulement un carnet pour garder en mémoire les itinéraires parcourus ${ }^{\prime}$ et sacrifier à la mode romantique du récit de voyage. Tenir le carnet fait en effet partie d'une discipline qu'Abbadie s'impose pour préparer un autre périple qui n'est que virtuel pour l'instant car outre l'observation des us électoraux des Londoniens, la description détaillée des architectures gothiques ou néo-gothiques d'Angleterre, d'Irlande ou d'Ecosse, outre les temps du voyage en bateau à vapeur et en malle-poste, parfaitement minutés et consignés ${ }^{2}$, existe, sousjacent, le voyage dans le voyage, mise en abyme présente de façon constante du début à la fin : l'Orient rêvé :

“(...) depuis que j'ai quitté Dublin, je n'ai pas ajouté un mot à mon journal. (...) Je l'ai dit à Burnes, je me le suis promis avant de quitter la France, je veux me préparer à tenir un autre journal dans ces lieux où il n'y a ni guides imprimés, ni autorités vivantes qu'on puisse consulter, où moi je dois tout voir où moi seul je dois tout noter. Le temps me presse : je vais donc reprendre à partir de Londonderry quitte ày. renouer le fil plus tard si je puis en retrouver les traces à Dublin.".

\section{Echos d'un Orient imaginaire :}

L'Orient est encore une contrée mal définie au début du XIX ${ }^{c}$ siècle, la formule "voyages en Orient "semble ne dater que de 1772 et l'usage au singulier de 1835 , précisément, date où Lamartine publie son livre, Souvenirs, impressions, pensées et paysages pendant un vovage en Orient."

Voyage long, coûteux et dangereux selon les destinations, nous verrons que le jeune A. d'Abbadie s'y prépare rigoureusement en cette même année 1835. Mais quoiqu'en filigrane la quasi totalité du joumal soit consacrée à ladite préparation, un Orient imaginé, rêvé apparaît çà et là tant à travers le texte du "magicien guérisseur" "que dans la "poésie arabe" .

Ce dernier texte, bien dans la veine orientaliste de l'époque est probablement de la main de d’Abbadie. Il y parle déjà de la violence du vent de Simoun. 
"Le vent du Simoun enlève des habits des voyageurs tous les autres parfums mais il n'enlève jamais du coeur l'odeur de cette fleur merveilleuse."

Antoine d'Abbadie a très cerainement été mis en garde par ses lectures sur les dangers de ce vent brûlant qu'il nomme encore de façon erronée "Simoun". La "brève" mais fort documentée notice qu'il lui consacre en 1873 dans "Géodésie d'Ethiopie", montre qu'il fait, pour la première fois, l'expérience du vent "empoisonné" en 1841 , lors de son séjour dans la mer Rouge. L'article de 1873 critique rétrospectivement, tout en les dévoilant, les sources livresques de 1835 : “Le samuwm (...) que nos lexiques ont tort d'appeler simoun".

La place importante accordée au "samuwm" dans la "poésie arabe", puis les explications détaillées données dans "Géodésie d'Ethiopie", fruits cette fois de l'expérience, témoignent de la véritable fascination exercée par ce vent sur l'imaginaire du voyageur et du scientifique Abbadie :

"Le vent mystérieux dit samuwum par les Arabes mérite une courte notice. On dérive ce mot de sumuwm pluriel de sim, poison, et à en juger par son action délétère sur l'économie animale, le samuwm serait une brise empoisonnée. (...) Le samuwm (...) ne dure que quelques minutes. Les Habab qui l'appellent harur, c'est à dire chaleur par excellence, disent que s'il dure un quart d'heure de suite aucun être humain ne survit à son terrible souffle; Du haut des montagnes, les pâtres voient alors les vovageurs et leurs bêtes rester immobiles là où ils se sont laissés tomber, et n'accourent que pour relever des cadavres.

Je n ai entendu parler du samuwm que dans les environs immédiats de la mer rouge, et seulement dans la saison chaude ou l'été boréal. Le 20 juillet 1841, à Muçoww'a. il venait de la terre ferme et sa température était égale à $46^{\circ} 5$, la plus forte chaleur de l'air que j'aie constatée en me préservant autant que possible des effets du ravonnement qui élève souvent ce chiffre. Il n'est pas étonnant que par une température pareille l'eau d'un bain à $36^{\circ} \mathrm{m}$ 'ait semblé tellement glaciale que je n'ai pas osé m'y plonger. Revenons au samuwm. Je l'ai observé à Addi Habib le 22 du mois suivant. Le sol s'était échauffé jusqu'à $60^{\circ}$, ce qui nous forçait à chausser nos sandales pour marcher sur un sol d'alluvion si doux qu'on préfere y cheminer à pieds nus. Nous étions réfugiés dans une hutte de nattes qui protégeaient for bien des ravons du soleil quand tout d'un coup le samuwm s'éleva pendant quelques minutes. (...)

Ce qui frappe le plus dans les phénomènes du samuwm c'est l'étonnante prostration de forces que ce vent amène dès qu'il se manifeste. En 1841 je caressais encore I'hypothèse qu'un phénomène météorologique peu commun se présentait toujours trois jours de suite. Le 23 septembre je m'attendais donc au samuwm pendant notre halte sur le terrain ouvert de Tarakaba. Après avoir choisi le moins mauvais ombrage, un acacia qui avait plus d'épines que de feuilles, je tirai mes thermomètres de leur étui et les plaçai sur une pierre à quelques décimètres de moi. L'effort à faire était minime. et cependant je ne pus l'effectuer quand le samuwm vint de la mer et, comme la veille. presque subitement. J'en appelai à toute l'énérgie de ma volonté pour dominer l'agonie si subite de mes forces musculaires, et quand $j$ 'y réussis enfin. le samuwm avait déjà cessé." "?

Le carnet de 1835 contient aussi, outre l'allusion aux Mille et une nuits qu'il fait relier à Bayonne ${ }^{8}$, la description imaginaire d'un château à l' architecture néo-gothique et de ses intérieurs. Le divan retiendra plus particulièrement notre attention : 
"Un divan à l'orientale éclairé par le haut et portant la nuit trois lampes, les murs tapissés de belle fä̈ence là où des tablettes sculptées ne portent pas de livres. Ceuxci sont tous romans naufrages et vovages gracieux. Un divan tout autour avec tapis de Turquie et une ou deux tables à échecs etc., entourent deux beaux houkas ${ }^{\text {shis. Un }}$ nègre sent le tabac à genoux."

Pour qui connaît le château d'Abbadia, un tel décor sera familier...

La "Sirène de Ceylan" (Mermaid of the island of Cevlon) qu'A. d'Abbadie voit un beau jour dans un bazar d'Edimbourg et qu'il prend soin de dessiner, vient, elle aussi, de cet Orient mystérieux aux frontières floues et lointaines. Le jeune scientifique sait bien, au fond, qu'il est face à un faux grossier, mais on sent bien qu’il préférerait croire à l'être fabuleux des légendes:

"La baroque figure ci-contre est dans le bazar à Edimbourg, avec l'inscription Mermaid of the island of Cevlon. Sa hauteur totale est d'environ Om5. La peau est desséchée et comme conservée dans l'alcool: il y a 4 nageoires sur le corps et une dorsale sur la queue. Si ceci est une fabrication comme c'est probable. du moins je ne puis en voir les joints." in

Mais A. d'Abbadie ne se complaît pas longtemps dans un Orient imaginaire ou des voyages vécus par procuration. Il veut, au contraire, dès bien avant 1835 , donner corps au désir d'explorer, vivre le voyage dans sa relation à l'espace, au temps, à son propre corps et à sa propre histoire par un entraînement physique régulier et une préparation d'une rigueur tout à fait spartiate. Et d'Abbadie a beau, comme tout voyageur, être parfois rassuré en retrouvant ailleurs des repères qui résonnent dans sa propre mémoire, seule l'intéresse, l'aire à cartographier, aire "vierge" à ses yeux de Blanc occidental vivant dans un siècle "d'aspiration au progrès" ".

\section{II "Voyager avec fruit" :}

\section{1. Arpenter l'inconnu :}

En 1867 Antoine d'Abbadie écrit :

"Quand il s'agit de vovager en pays nouveau et relativement inconnu, on veut avant tout savoir jusqu'où le vovageur est allé, les directions et les distances des lieux qu'il a visités, enfin leur altitude. qui donne de prime abord une idée très approchée du climat. Ces résultats sont les premiers qu'on demande à l'explorateur dès qu'il est revenu dans sa patrie"

Ces lignes ont été rédigées trente-deux ans après la rédaction du carnet qui nous occupe : pour lors, d'Abbadie a réalisé une partie des explorations rêvées, il été en Egypte, (1836/1837, 1848), il a exploré la mer Rouge (1839) ${ }^{12}$ puis l'Ethiopie (1840 à 1848)...Cela ne l'empêche pas de se référer encore à la genèse de ses voyages et notamment aux conseils prodigués par Mac Gregor Laird dans le camet de 1835.

Un article rédigé bien des années plus tard nous apprend qu'Antoine d'Abbadie projette d'aller en Afrique dès 1929 et qu'il s'y est préparé assidûment jusqu'en cette année 1835 :

"Avant formé au sortir du collège, en 1829. le projet d'une exploration dans l'intérieur de l'Afrique où je voulais alors entrer par Tunis ou le Maroc. je consacrai une grande partie des six années suivantes à étudier les sciences nécessaires pour vovager avec fruit." !3 
Des "Notes autobiographiques" rédigées par Abbadie sur le tard et encore inédites ${ }^{1 \downarrow}$ complètent le portrait ébauché ci-dessus puisque nous le voyons après 1832 , date du décès du père, menant une vie de "complète solitude" à Paris avec le reste de sa famille et étudiant sans relâche pour préparer ses futurs voyages :

"Je passais", dit-il, "mes journées soit aux cours de la Sorbonne, soit dans un laboratoire savant où je travaillais satisfaire cette passion des voyages qui me hantait depuis mon enfance"

Antoine d'Abbadie est, à l'époque, un jeune homme introverti "s, studieux et volontaire, habité par une passion et qui étouffe probablement au sein d'une société trop bourgeoise et corsetée. Le goût pour le nomadisme ne lui vient-il pas aussi de famille, lui, dont la mère est Irlandaise, qui est né en Irlande et dont le père basque "commis chez un négociant français à Séville", puis promu négociant a dû lui faire le récit de ses propres déambulations ? Les mêmes "Notes autobiographiques", décidément bien précieuses, nous apprennent, au passage, que le père d'Antoine avait quitté la France avant la Révolution ${ }^{16}$ et non par la suite :

"Mon aïeul aimait l'ancien régime, mais pressentant la Révolution, il envova en Espagne son fils Amaud Michel. C'est ainsi que mon père entra comme commis chez un négociant français à Séville. $s$ 'enrichit par des conseils et devint son associé. Tout marchait à souhait quand le Roi d'Espagne irrité après la mort de Louis XVI, ordonna à tous les français, alors nombreux, de se faire Espagnols ou de quitter ses Etats dans les 24 heures. Mon père s'embarqua aussitôt pour l'Angleterre et s'y associa avec un négociant protestant établi dans la ville de Hull. (...)

Livré à ses propres ressources et privé par la mort de son premier associé à Séville. mon père panint néanmoins à amasser une fortune. Il alla en Irlande et s'y maria." 17

Les heures passées en bibliothèque ne forment qu'une partie de l'entraînement ; trois années plus tard, date à laquelle il écrit son joumal, nous pouvons constater que les exercices physiques sont intenses : courses par tous les temps en Béarn, aux environs du château d'Audaux, en Soule, sur la Côte Basque et en particulier au-dessus des falaises entre Socoa et Hendaye ${ }^{18}$.

Cet entraînement physique a vraisemblablement dû commencer bien avant 1835 , dès la décision d'aller en Afrique prise. Le journal de 1835 dévoile aussi que I'entraînement suivi ne se limite pas à tester et à augmenter l'endurance. Il est indissociable de l'étude topographique : mesurer la largeur d'une rivière, la hauteur d'une montagne, et se complète par des exercices et des calculs "in situ" réalisés à l' aide de divers instruments et lunettes ou à l'oil nu, ainsi ceux réalisés (probablement à Bordaberri à Urrugne) avec l'aide de Domingo son domestique :

"J'ai mesuré une base de $500 \mathrm{~m}$. dans ma prairie et me plaçant de façon à voir Domingo projeté sur un mur couver de lierre et distant de $1000 \mathrm{~m}$. $j$ 'ai fait les observations suivantes à l'oeil nu.

A $500 \mathrm{~m}$. je vois la couleur tranchante des vêtements et le mouvement de la marche même quand il a lieu dans la direction du ravion visuel. En repos je vois à peine la personne. Un cheval perd tout le moelleux des formes. Un cri médiocre s'entend assez bien quand il va avec le vent mais faisant un angle de $20^{\circ}$ contre le vent je ne me ferais pas entendre à cette distance en criant de toutes mes forces; 
A $400 \mathrm{~m}$. tout ceci est un peu mieux défini, mais il n'est rien de bien tranché

A $300 \mathrm{~m}$. je sépare le visage du corps je vois le mouvement des vétements quand ils sont poussés légèrement par le vent. (...)

Avec la lunette ayant le diaphragme:

A 500 m. le visage est blanc, confus sans traits : les doigts même ouverts ne se distinguent pas. (...)" ${ }^{\text {"i }}$

En voulant rendre connu ce qui est inconnu, en désirant mesurer, répertorier, cartographier les terres du Levant, A. d'Abbadie prend part au vaste mouvement de "célébration collective" qui porte l'occidental du XIX" à affimer son ordre culturel". Ceci dit, la manière de voyager de d'Abbadie doit beaucoup au siècle qui l'a précédé et notamment à un philosophe des Lumières comme Diderot qui conseille comment "vovager utilement":

"Je voudrais au voyageur une bonne teinture de mathématiques, des éléments de calcul, de géométrie, de mécanique, d'hydraulique, de physique expérimentale, d'histoire naturelle, de chimie, de dessin, de la géographie, et même un peu d'astronomie; ce qu'on a coutume de savoir à vingt deux ans, quand on a reçu une éducation libérale.

(...) Que la langue du pars ne lui soit pas tout à fait inconnue; sil ne la parle pas. du moins qu'il l'entende.

Avez lu tout ce qu'on aura publié d'intéressant sur le peuple que vous visiterez. Plus vous saurez, plus vous aurez à vérifier, plus vos résultats seront justes. " "I

L'auteur du carnet est bien un jeune homme dans l'esprit de Diderot, son voyage en Orient est préparé dans les moindres détails par une solide formation dans les disciplines essentielles que sont pour tout explorateur les mathématiques, l'astronomie, la physique, la géographie, l'histoire, la technique, l'étude des langues (le carnet donne la preuve que d'Abbadie est en train d'étudier l'arabe en 1835). Cela ne l'empêche pas, outre les entraînements physiques dont nous avons parlé, de consigner les précieux conseils glanés auprès des explorateurs Mac Gregor Laird, Burnes ou Cailliaud. Ces conseils lui seront utiles fort longtemps puisqu' il ne cessera, par la suite, de les compléter par des observations personnelles et d'y ajouter les récits de voyage d'autres explorateurs comme, par exemple M. Palgrave 22 .

Les maîtres mots de tous ces conseils sont l'adaptation et la prudence.

\section{2. S'adapter et ouvrir l'œil :}

Il faut tout d'abord s'adapter au climat, au terrain, aux moyens de communication. Abbadie mentionne souvent, dans son carnet, les "causeries" de Burnes et notamment ses observations sur le voyage à dos de chameau :

"Pour connaître la marche d'un chameau il mesurait le terrain occupé par une dizaine de ces hêtes attachées à la file l une de l'autre. puis le temps qu'elles mettaient à le parcourir." :

Le scientifique n'est rien sans ses instruments de mesure, il s'agira donc de les adapter à la situation. Peut-on les porter sur un chameau ?

"M. Tiarks croit qu 'il est bon de porter un chronomètre même sur un chameau." 24

Il est évident qu il faudra les modifier, les rendre pliables ou plus petits ("une règle en cuivre entrant dans le portefeuille") "s, plus légers afin qu'ils soient aptes au transport. Abbadie qui inventera lui-même plusieurs instruments scientifiques insiste sou- 
vent sur la nécessité de voyager léger, d'emporter le minimum nécessaire et il établit, pour ce faire, une liste détaillée de "l'attirail de petit vovage", de "grand vovage", de même que ce qui est nécessaire "pour une course rapide à pied" 26

Autre nécessité : adapter son habillement au climat. Abbadie recopie précieusement dans le carnet la lettre en anglais de l'explorateur du Niger Mac Gregor Laird (de même qu'il note tout aussi précieusement les conseils donnés par Cailliaud) ${ }^{27}$, lettre qu'il traduit intégralement plus de trente ans plus tard dans les "instructions pour les voyages d'exploration" :

"Si vous avez pris l"habitude de porter flanelle, continuez à le faire; sinon, ne vous y mettez point. Une couverture ordinaire en laine est le préservatif le moins cher et le plus sûr contre la chaleur ou le froid. Une ceinture de laine ou même de coton, portée au creux de l'estomac, prévient la dvsenterie. Tenez votre tête rasée et bien couverte. Ne portez jamais de bas et usez de souliers le plus rarement possible. Adoptez I'habillement indigène en tant qu 'il vous semblera commode; ne vous déguisez jamais en tâchant de passer pour un indigène." 28

Ceci nous amène à l'autre corollaire : la prudence. Prudence dans l'alimentation, Mac Gregor Laird conseille de manger le moins possible, de "ne jamais boire de l'eau pure, mais bien mélangée de farine ou d'un peu d'eau de vie" et de ne jamais user de viande rôtie mais bouillie ${ }^{2}$. Car le grand danger c'est la maladie : dysenteries, ophtalmie (dont Abbadie souffrira beaucoup), constipations, hémorroïdes. Abbadie note, tout d'abord pour lui-même en anglais en 1835, puis en 1867 en français pour les lecteurs français, les remèdes indiqués par Mac Gregor Laird :

"Si vous reconnaissez l' invasion de la fièvre par la douleur ou un serrement autour des tempes, des maux de coeur et une forte chaleur aux mains et aux pieds, prenez deux grains (un décigramme) d'émétique et deux cuillerées de sulfate de magnésie. mélés avec autant d'eau tiède que vous pous pourrez en avaler." 30

Parmi les maladies redoutables : celles sexuellement transmissibles comme la terrible syphilis ou la blennoragie citées dans le carnet de 1835 , puis à nouveau dans l'article de 1867 :

"On guérit les hémorroüdes et la blennorragie par des compresses d'eau froide souvent renouvelées \& portées pour le ler cas, peu à peu, à la température de $0^{\circ} . " 31$

"Un homme qui s'est fait connaître comme chirurgien \& que j'ai vu au Havre me dit qu'une fermme avait apporté de l'Amérique du Sud une maladie vénérienne telle qu'au bout de 36 heures le pénis et les testicules se détachaient du corps sans que du reste la santé générale en souffrît notablement. Quelqu' un présent cita à cet effet une maladie vénérienne du Portugal connue sous le nom de Yaws et qui est dit-il, terrible" ${ }_{32}$

"Les femmes sont à redouter ".sus dit Abbadie en 1835, puis en 1867, revenant sur le conseil de Mac Gregor Laird: "Be virgin" "3n, i] ajoute :

"La règle d'Horace s'applique au voyageur tout aussi bien qu'à l'athlète : abstinuit venere et vino. C'est avant de partir et en Europe même qu'on doit apprendre par la pratique ses limites personnelles pour tous les exercices du corps, pour l'abstinence. le jeûne même, et pour la faculté de supponer la soif el le manque de sommeil. En voiage on devra toujours rester bien en deçà de ces limites. "..

En cette année 1835, Antoine d'Abbadie, vingt cinq ans, projette depuis longtemps déjà de quitter l'Europe et de se diriger vers l'ailleurs. vers un Orient lointain qui l'at- 
tire irrésistiblement. Il croit le départ proche, il devra cependant d'abord obeir au Professeur Arago qui l'envoie en 1836 en mission au Brésil avant d'embarquer le $1^{\circ}$ octobre 1836 à Marseille pour l'Egypte.

Le "deuxième dimanche" après son départ, Le Caire lui apparaît "telle que Fénelon l'a dépeinte, presque aussi hasse que la mer" "s.

Désormais, il pourra juger par lui-même.

\section{A. ARKOTXA-SCARCIA UPRESA du CNRS}




\section{NOTES}

1. Pour avoir davantage de détails sur les itinéraires figurant dans le camel de 1835, cf A. ArkolxaScarcia "Les itinćraires d"Antoine d'Abbadie : annéc 1835", in Antoine d'Abbadie Pensées Etudes et Vorager de 1835 (camet inédit) tome 1. Ed. Patri Urkizu, 1997.

2. En ce qui concernc les caractéristiques du voyage de 1835 (lemps. paysages ruraux et urbains. architccturcs, habitants...). cf. A. Arkotxa-Scarcia "1835eko A. Abbadiaren bidaietaz" in Actes du colleque sur A. d'Abbadic (a paraitrc).

3. A. d'Abbadic, Penséer, études el vovages de 1835 . p. 81

4. Cr. J.C. Bcrchet. Lo vovage en Orient, anthologie des voyageur. franfais dans le Levant all XIX siecle. R. Laffont, 1985, p. 4.

5. Cr. lanalyse qu'en fait. dans cc même numéro, Y. Cardaillac-Hermosilla. "Lc magicien guérisseur du canct de voyagc de 1835 d'Antoine d' Abbadic." Le texte pourra être consulté en anjexe I du même articlc ou in Pensées études el vovager de $1835 \mathrm{~d}^{\circ} \mathrm{A}$. d Abbadic, pp.123/124 où I'on remplacera "owictan". crreur de transcription, par "orvietan" qui est lc terme appropric.

6. Cr.A. d'Abbadic, in op. cit.p. 105, of aussi remarques apportées par Y. Cardaillac-Hermosilla in op. cit. de mêmc que le texte du pod̀mc en annexe 2.

7. A. d Abbadic. "Géodésie d'Ethinpie", in Recucil de textes, tome 2, Ed. Urkizu 1997.

8. Cf. Pensées études et vovages de $1835, \mathrm{p}, 121$.

8 bis. Houka : pipe orientale analogue au narguile.

9. In op. cil. p. 108. Cr. aussi p. 107.

10. In op. cit. p. 85.

11. A. d'Abbadic "Notes pour voyager avec fruit", in Recueil de textes, tome 2, Ed. Urkizu p.167)

12. "Les armes et les savants de la France nous semblent n'avoir rien laissé à dire sur la Syrie et l'Egyple. La Mer Rouge seule est restée dans les ténèbres de l'éloignement. Nous voulâmes étudier ses parages. dont les destinées ćcillent l'anxiété légitime de toute l'Europe, el, après une excursion sur ses rivages orientaux, chez ces tribus arabes encore si peu connues, nous sommes allér vers l Occident vistier ces peuples d' Ethiopies qui s'étendent nystérieusement jusqu 'aux contres centrales de l'Afrique. " (1839, "Souvcnirs d'un voyage dans le sud" in op. cit. p.84)

13. A. D' Abbadic "Gédésie d'Ethiopic", in Recueil de textes.... Ed. Patri Urkizu tome 2, 1997 p. 220.

14. Cf. article de J.B. Orpustan : "Les noms de maisons issus du latin abbatia dans lc Pays Basquc médieval..." in Actes du colloque sur A. $d$ 'Abbadie (à paraître).

15. Voici par excmiple comment Antoinc définit les differences psychologiques ct donc de comportement qui le séparent d'Amauld :

"On sail assez la différence d"esprit qui existe souvent même entre frìres. Né pour commander le mien prenait son parti rapidement et s'exprimait d'ordinaire sur un ton qui $n$ admentait pas la contradiction. Il élait loul simple que par sa mqnière de parler et dagir il façonndı son enourage. mêne sans le voulloir. à cette pente de son esprit. La nienne était toute différente au lieu de surmonter hardiment lobstacle je trouvais qu' il ćtait plus facile de le tourner; cédant en apparence. je persévérais toujours et parvenais d force de patience a gagner le mème avantage que mon frère oblenait de prime saut. Fallail-il tenter une. route évidemnent dangereuse? Il annonçait son projet hautement et si longtemips d avance, que les partis armés qui interceptaient le chemin renonçaient a le faire devant une volonté trop bien affirmée pour ne pas la supposer étavée de movens materiels suffisants, ou bien parce qu ils ne crovaient plus a un vovage loujours remis au lendemain. Je partais au contraire si subitement que les voleurs de grand chemin $n$ 'avaient par le lenps d'être prévenus." (Recucil de lexıer. Lome 2, Ed. Urkizu 1997. pp. 300. 301)

16. M'élant fiée aux sources hahituelles cı n'ayant pas cu connaissance de ce dernier texte de d'Abbadic Iorsque je rédigcais l'avant-propos des Pensées. études et vovages de $1835 \ldots$ intitulé "Les itincraircs d'Antoinc d'Abbadic : anncec 1835". j'ai, moi aussi fail la même crreur en Eccrivant qu" "Arnaud Michel." (avail) "émigré à Londrer en 1793, sous la Terreur..." (in op. cit.. p. 30). Cf. aussi à ce propos l'article de J.B.Orpustan "Les noms de maisons issucs du latin abbatia dans le Pays basque médiéval (Labourd. BasscNavarte et Soule)" in Actes du colloque sur A d'Abbadic (à paraîtrc)

17. Cr. aricic de J.B.Orpustan in op. cit

18. Cf. A. Arkolxa - Scarcia "1835eko A. Abbadiaren bidaictaz" in Actes du colloque sur A. d Abbadie (a paraitre). 
19. A. d'Ahbadic: Pensées études et vovages de $1835 \ldots ., 127$.

20. Cr. J.C. Bcrchct. Le vorage en Orient... p. 12.

21. Denis Diderol "Voyager utilement" cite par J.M. Goulemot. P. Lidsky et D. Masseau in Le vovage en France. Anthologic des voyagcurs européens en France, du Moyen Âge à la fin de l'Empirc, Robert Laffont 1995. p. 517.

22. Cr.A. d'Abbadic "L'Arabic. ses habitants. leur ćtat sociaj et religieux, d' après la relation du voyagc de M. Palgrave". in Recueil de texzes p. 127.

23. A. đ’Abbadic. Pensées études et vovages de 1835. p. 52

24. In op cit p. 62.

25. In op cit p. 132.

26. In op. cit. p. 106/107.

27. In op cit p. 134.

28. In Recueil de textes... p. 180.

29. In op cit p. 181

30. In op cit p. 181.

31. Pensées, éludes et vorages de $1835 \ldots$ p. 106.

32. In op cit p. 110.

32 bis. In op cit p. 86 .

33. in op cil p. 103

34. In op cil p. 179

35. A. d'Abbadie, "Lettre à un ami", in Recueil de textes p. $87 / 88$ 